A-32-2020

\title{
ESTUDIO DE LAS RELACIONES HÍDRICAS EN CEREZOS SOMETIDOS A CICLOS DE SUPRESIÓN - RECUPERACIÓN DEL RIEGO
}

\author{
Blaya Ros, P.J. ${ }^{1}$, Blanco Montoya, V. ${ }^{1}$, Torres Sánchez, R. ${ }^{2}$, González Teruel, J.D. ${ }^{2}$, Soto \\ Valles, F. ${ }^{2}$, Toledo Moreo, A.B. ${ }^{2}$, Jiménez Buendía, M. ${ }^{2}$, Domingo Miguel, $R^{1}{ }^{1}$
}

${ }^{1}$ Dpto. de Ingeniería Agronómica, Universidad Politécnica de Cartagena, Paseo Alfonso XIII, 48. 30203, Cartagena. pedro.blaya@upct.es; victor.blanco@upct.es; rafael.domingo@upct.es ${ }^{2}$ Dpto. de Automática, Ingeniería Eléctrica y Tecnología Electrónica, Universidad Politécnica de Cartagena (ETSII-UPCT). roque.torres@upct.es; juando.gonzalez@upct.es; pencho.soto@upct.es; ana.toledo@upct.es; manuel.jimenez@upct.es.

\section{Resumen}

En climas áridos y semiáridos, donde los recursos hídricos son escasos, es necesario aumentar la eficiencia en el uso del agua. Para ello, el conocimiento de las relaciones hídricas se convierte en un factor determinante. Con el objetivo de estudiar la respuesta fisiológica y vegetativa de cerezos jóvenes (Prunus avium L.) "Lapins" se realizó un experimento en 2019 consistente en la aplicación de dos ciclos de supresión - recuperación del riego a partir de tres tratamientos: i) control, CTL, regado al $115 \%$ de sus necesidades hídricas máximas durante todo el ensayo; ii) déficit moderado-severo, MS, regado como CTL hasta finales de junio, momento a partir del cual se alternaron 2 ciclos consecutivos de supresión - reanudación del riego; iii) déficit severo, SS, similar a MS pero con árboles sometidos a un estrés de mayor intensidad y duración. Los indicadores de suelo y planta estudiados fueron sensibles al estrés hídrico, lo que provocó la caída de los potenciales matricial del agua en el suelo e hídrico de tallo a mediodía, y un aumento de la temperatura de la copa y de los índices derivados de ésta. Todos los indicadores estudiados alcanzaron valores similares a CTL durante los periodos de recuperación. El crecimiento vegetativo se vio drásticamente afectado por la intensidad y duración del déficit hídrico.

\section{1) Introducción}

La cuenca mediterránea caracterizada por un marcado déficit hídrico estacional, acentuado como consecuencia del cambio climático, es un área donde el uso eficiente del agua es clave. En estas zonas, caracterizadas por tener un clima semiárido y árido, se producen frecuentes episodios de sequía, los cuales ponen en peligro al principal consumidor de los suministros hídricos, la agricultura (Fereres y Soriano, 2007). Esta realidad, cada vez más agravada, ha hecho necesario incrementar la eficiencia en el uso del agua y realizar una programación óptima del agua disponible, sin afectar a la producción o calidad de la cosecha.

La programación precisa del riego puede contribuir a ello, a través del conocimiento directo o indirecto del estado hídrico de la planta a partir de distintos indicadores. Existen númerosos indicadores del estado hídrico del suelo y de la planta de utilidad en la elaboración y supervisión de programas de riego, si bien, son los de suelo los más frecuentemente utilizados a nivel comercial, debido a que sus medidas pueden ser fácilmente automatizables (Jones, 2007) e interpretables. Sin embargo, el número de medidas necesarias para representar adecuadamente el estado hídrico del cultivo suele ser superior a los basados en planta ya que a pesar de su alta intensidad de señal se ven afectados por la por la alta variabilidad de las medidas (Naor et al., 2006). En contraste, los indicadores del estado hídrico de la planta, aunque representan con mayor fidelidad su estado hídrico, son aplicados principalmente en el ámbito de la investigación por su mayor dificultad de medida e 
interpretación. Tradicionalmente los investigadores han sugerido el uso del potencial hídrico de tallo como el indicador de referencia para determinar el estado hídrico de la planta (Schackel et al., 1996); sin embargo, su aplicabilidad en campo es compleja, debido a que es una técnica muy laboriosa y no automatizable. Otro indicador de planta que se está imponiendo en los últimos años es la temperatura de copa, Tc, debido al desarrollo de equipos capaces de realizar medias continuas y a distintas escalas, es decir, desde una hoja hasta una cuenca (García-Tejero et al., 2018a). La regulación de Tc es de suma importancia para las plantas, debido a que pequeñas modificaciones puede afectar de forma contundente a procesos fisiológicos importantes en los que intervienen reacciones químicas, y los relativos a la división y el crecimiento celular, los cuales pueden verse gravemente afectados a temperaturas extremas (Jones, 2018).

\section{2) Objetivo}

El objetivo del presente trabajo fue estudiar la respuesta fisiológica y vegetativa de cerezos jóvenes sujetos a dos ciclos de supresión - recuperación del riego, con el fin de valorar la utilidad de las medidas de temperatura de copa y los índices derivados de ella para detectar y cuantificar situaciones de estrés hídrico en planta y su interés para el manejo del riego.

\section{3) Material y Métodos}

El ensayo se llevó a cabo en la Estación Experimental Agroalimentaria 'Tomás Ferro' (ETSIA-UPCT), ubicada en Cartagena, durante el periodo junio - septiembre de 2019 (días del año, DDA, 170 - 268). El experimento se realizó en cerezos (Prunus avium L.) "Lapins" de 3 años de edad, injertados sobre "Mirabolano", y a marco de 3,5 m x 2,25 m. Cada árbol se regó a partir de tres emisores autocompensantes de $2,2 \mathrm{~L} \mathrm{~h}^{-1}$, colocados en un único lateral de riego y a $75 \mathrm{~cm}$ de separación. El agua de riego, proveniente del transvase Tajo-Segura, presentó una conductividad eléctrica $\left(\mathrm{CE}_{25^{\circ} \mathrm{C}}\right)$ de $1,1 \mathrm{dS} \mathrm{m}^{-1}$.

Al inicio del ensayo se establecieron 3 tratamientos de riego según un diseño de bloques al azar con 3 repeticiones y 4 árboles por repetición: i) CTL, control, regado para satisfacer el $115 \%$ de las necesidades hídricas del cultivo, ii) MS, déficit moderado-severo, regado como CTL hasta finales de junio, momento a partir del cual se alternaron 2 ciclos consecutivos de supresión-reanudación del riego. El riego se reanudó tras alcanzar un potencial hídrico de tallo, $\Psi$ stem, de -1,3 MPa en el primer ciclo y de -1,6 MPa en el segundo; y iii) SS, déficit severo, similar a MS, pero con recuperaciones a -1,6 MPa y -2,3 MPa. Los periodos de recuperación se dieron por finalizados cuando los valores de $\Psi$ stem de los tratamientos deficitarios alcanzaron valores de $\Psi$ stem similares a los de los árboles CTL durante al menos 4 días consecutivos.

Las variables meteorológicas fueron proporcionadas por la estación climática CA12 del Servicio de Información Agraria de la Región de Murcia, SIAM, ubicada a $200 \mathrm{~m}$ de la parcela experimental. Las condiciones meteorológicas fueron típicas del clima mediterráneo con veranos secos y calurosos e inviernos suaves y húmedos. Las necesidades hídricas del cultivo fueron calculadas semanalmente a partir de la evapotranspiración del cultivo, ETc, según la metodología FAO:

$$
\mathrm{ETc}=\mathrm{ET}_{0} \times \mathrm{Kc} \times \mathrm{K}_{\mathrm{L}}
$$

donde, $\mathrm{ET}_{0}$ es la evapotranspiración de referencia (Allen et al., 1998); Kc, coeficiente de cultivo (Marsal, 2012); KL, coeficiente corrector por localización dependiente de la superficie del suelo cubierta por las copas de los árboles (Fereres y Castel, 1981).

Con el objetivo de conocer el estado energético del suelo de forma continua se instalaron tres sensores (MPS-6 Decagon Devices, Pullman WA, USA) por tratamiento de medida dieléctrica, calibrados y con compensación térmica para monitorizar el potencial matricial de agua en el suelo, $\Psi \mathrm{m}$. Los sensores fueron colocados a $25 \mathrm{~cm}$ de profundidad y 
a $23 \mathrm{~cm}$ del emisor central. El análisis de los datos $\Psi \mathrm{m}$ se realizó a partir de la media de los valores registrados en el periodo de 8:00-10:00 h UT.

El estado hídrico de la planta fue determinado a partir del potencial hídrico de tallo, Ystem, y de la temperatura de copa, Tc, medidos a mediodía (11:00 - 13:00 UT) cada 3-7 días. Ystem fue determinado con una cámara de presión tipo Scholander (mod. SF-PRES70, SolFranc Tecnologías, S.L., Spain) en 2 hojas adultas, sanas y cercanas al tronco por repetición $(n=6)$. Tc fue medida con una cámara térmica (Flir One, Flir Systems, Wilsonville, OR, USA), conectada a un teléfono inteligente, smartphone. Se tomaron dos imágenes por repetición $(n=6)$ en la parte soleada del árbol y a una distancia de 1,5 $\mathrm{m}$ de la copa, de acuerdo con las indicaciones propuestas por Costa et al. (2013) e Idso et al. (1981). La emisividad fue configurada en "matt" ( $\varepsilon=0,95$; García-Tejero et al., 2018b) y las imágenes fueron analizadas en el programa informático Flir Tools application (Flir One, Flir Systems, Wilsonville, OR, USA), siguiendo las recomendaciones propuestas por Gómez-Bellot et al. (2015)

Una vez obtenida Tc, y para mitigar el efecto de las variables meteorológicas, dos índices térmicos fueron calculados: (i) la diferencia entre la temperatura de la copa y la del aire circundante $(\Delta T)$; y ii) el índice de estrés hídrico del cultivo (CWSI, crop water stress index; Jackson et al., 1981):

$$
\begin{aligned}
& \Delta \mathrm{T}=\mathrm{Tc}-\mathrm{Ta} \\
& \text { CWSI }=\frac{\Delta \mathrm{T}-\Delta \mathrm{T}_{\text {wet }}}{\Delta \mathrm{T}_{\text {dry }}-\Delta \mathrm{T}_{\text {wet }}}
\end{aligned}
$$

donde, Ta es la temperatura del aire circundante en el momento de la medida; Tc es la temperatura de la cubierta vegetal; $\Delta \mathrm{T}_{\text {wet }}$ es la diferencia entre la temperatura de la copa y la del aire circundante cuando el cultivo tiene los estomas completamente abiertos y $\Delta$ $T_{\text {dry }}$ cuando están completamente cerrados. De acuerdo con Jones (1999), $\Delta T_{\text {wet }}$ fue determinada a partir de la línea límite inferior, $\mathrm{LLI}\left(\mathrm{T} \Delta_{\text {wet }}=\mathrm{a}+\mathrm{b}^{*} \mathrm{DPV}\right)$, obtenida tras la pulverización del limbo de la hoja con una solución de agua y detergente al 0,01\% (v/v) aproximadamente $1 \mathrm{~min}$ antes de realizar la imagen. $\Delta \mathrm{T}_{\text {dry }}$ fue determinada a partir de la línea límite superior, LLS. Para provocar un cierre estomático total y obtener LLS, se aplicó una fina capa de petroleum jelly (vaselina) en el limbo $1 \mathrm{~h}$ antes de la medición. Los datos de Ta, humedad relativa (HR) y el déficit de presión de vapor (DPV) fueron obtenidos mediante tres sensores climáticos (VP-4, Decagon Devices, Pullman WA, USA) y almacenados en un datalogger (CR1000, Campbell Scientific Ltd., Logan, USA).

Adicionalmente, se evaluó el crecimiento vegetativo del cultivo a partir del incremento del diámetro de tronco, $\Delta \mathrm{DT}$, y del peso de la poda de invierno. $\triangle \mathrm{DT}$ fue medido con una cinta métrica (Pi meter MF612 A, Weiss, Erbendorf, Germany) y los pesos de poda con una balanza electrónica (Scaltec, modelo SSH91, Heiligenstadt, Germany) en los dos árboles centrales de cada repetición $(n=6)$.

El análisis estadístico de los datos se realizó con el programa informático SPSS Statistics (SPSS Inc., 24.0 Statistical package; Chicago, IL, USA), a partir del análisis de la varianza (ANOVA) y pruebas de comparación múltiples a un nivel de confianza del $95 \%$. Las regresiones lineales y no lineales entre los indicadores fueron realizadas con Sigmaplot Plus v.12.5 (Systat Software, San Jose, CA, USA). 


\section{4) Resultados y Discusión}

El indicador $\Psi m$ evidenció el régimen hídrico impuesto por los ciclos de supresiónreanudación del riego (Figura 1). Los árboles del tratamiento CTL mantuvieron, durante todo el periodo estudiado, valores medios de $\Psi \mathrm{m}$ característicos de capacidad campo $(\Psi \mathrm{m}>-30$ $\mathrm{kPa}$ ) y semejantes a los mostrados por Blanco et al. (2018) en cerezos adultos y sin restricción hídrica. Por otro lado, los árboles de los tratamientos deficitarios, MS y SS, mantuvieron valores similares a CTL en los periodos en los que se satisficieron las necesidades hídricas. Tras la supresión del riego en el tratamiento MS, Ym disminuyó de forma abrupta hasta alcanzar valores mínimos de $-744 \mathrm{kPa}$ (DDA 186) y -814 kPa (DDA 221) durante el primer ciclo y segundo ciclo de supresión, respectivamente. Por su parte, SS, tratamiento sometido a un mayor estrés hídrico en planta, mostró valores mínimos de $-540 \mathrm{kPa}$ (DDA 197) y -670 $\mathrm{kPa}$ (DDA 235) durante el primer y segundo periodo de supresión, respectivamente. A pesar de que el segundo periodo de restricción hídrica en los árboles SS fue superior (DDA 208 242), no se alcanzaron valores mínimos similares a MS, debido a las precipitaciones acaecidas (19 mm) entre los DDA 233-240, lo que provocó un aumento de los valores $\Psi \mathrm{m}$ y, por lo tanto, una recuperación parcial del estado energético del agua en el suelo. En ambos tratamientos deficitarios se observó un cambio de pendiente en la caída de $\Psi \mathrm{m}$. La ralentización de la pendiente, asociada al agotamiento del agua en suelo, fue de mayor duración en el tratamiento SS. La alta variabilidad de los valores $\Psi \mathrm{m}$, suele ser debida a la heterogeneidad del suelo y a la distribución del sistema radical (Naor et al., 2006).

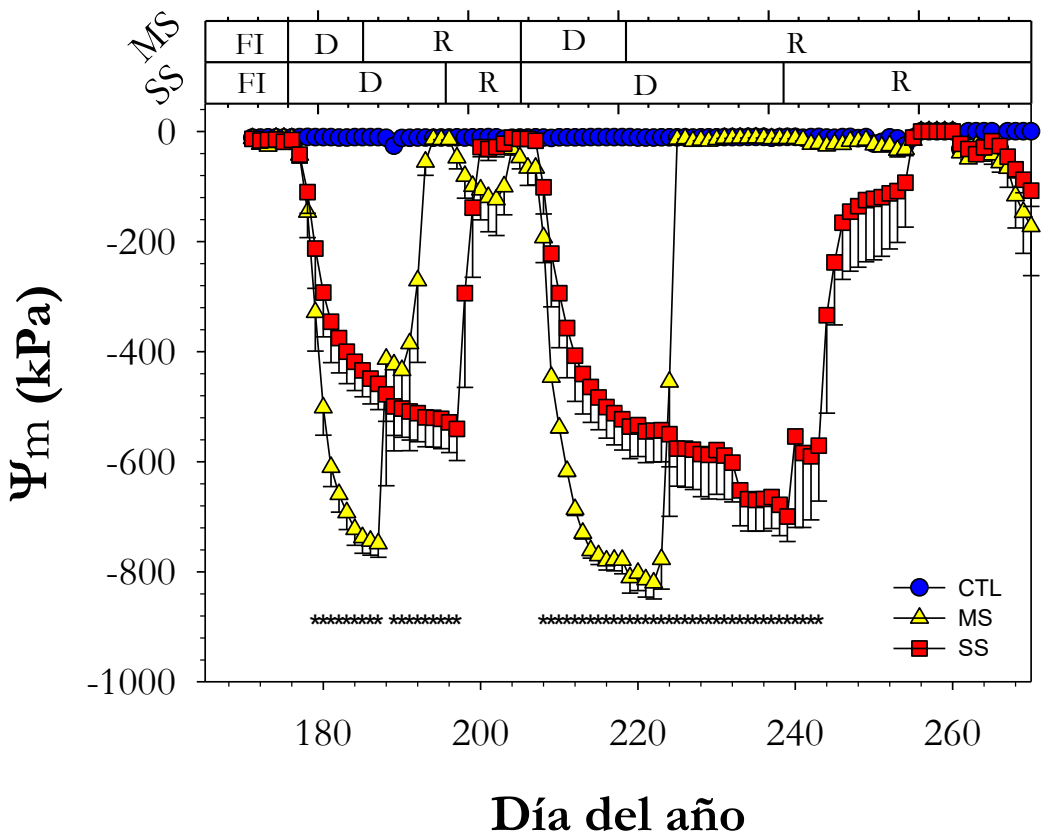

Figura 1. Evolución del potencial matricial del suelo, $\Psi \mathrm{m}$, durante el periodo de estudio. Cada punto es la media \pm el error estándar (ES) de 3 medidas por tratamiento. Los asteriscos indican diferencias significativas según el test de Rango Múltiple de Duncan $(p<0,05)$. Las barras horizontales superiores indican los periodos de los ciclos supresión-recuperación del riego que definieron a los tratamientos MS y SS. 'Fl' es el periodo previo al inicio de los ciclos de supresión-recuperación, en el que ambos tratamientos fueron regados como CTL; ' $D$ ', periodo de supresión de riego; ' $R$ ', periodo de recuperación de los árboles.

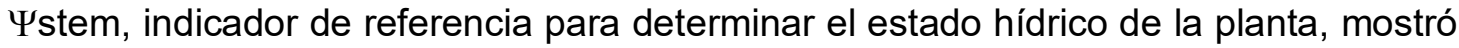
una tendencia parecida a $\Psi \mathrm{m}$ (Figuras 1 y 2a), viéndose fuertemente influenciado por la supresión del riego. El tratamiento CTL mantuvo valores $\Psi$ stem propios de árboles sin limitaciones hídricas ( $\Psi$ stem medio $=-0,62 \mathrm{MPa}$ ) y similares a los obtenidos por Blanco et al. 
(2019) en cerezo. Los valores de $\Psi$ stem de los árboles deficitarios, una vez que el riego fue suprimido, disminuyeron gradualmente hasta alcanzar los valores umbrales $\Psi$ stem preestablecidos. Los valores mínimos de $\Psi$ stem alcanzados tras la supresión del riego fueron $-1,24 \mathrm{MPa}$ y $-1,86 \mathrm{MPa}$ en el tratamiento MS y -1,64 MPa y -2,40 MPa en el tratamiento SS para el primer y segundo ciclo, respectivamente. Blanco et al. (2018) reportaron como perjudiciales valores $\Psi$ stem inferiores a -1,3 MPa en cerezo "Prime Giant", ya que suponen un agotamiento importante de las reservas hídricas de las ramas principales, y, por lo tanto, de su capacidad de contracción diaria. En idéntica línea, Marsal et al.,(2010) consideran que valores de $\Psi$ stem inferiores a -1,5 MPa pueden perjudicar significativamente a producciones futuras.
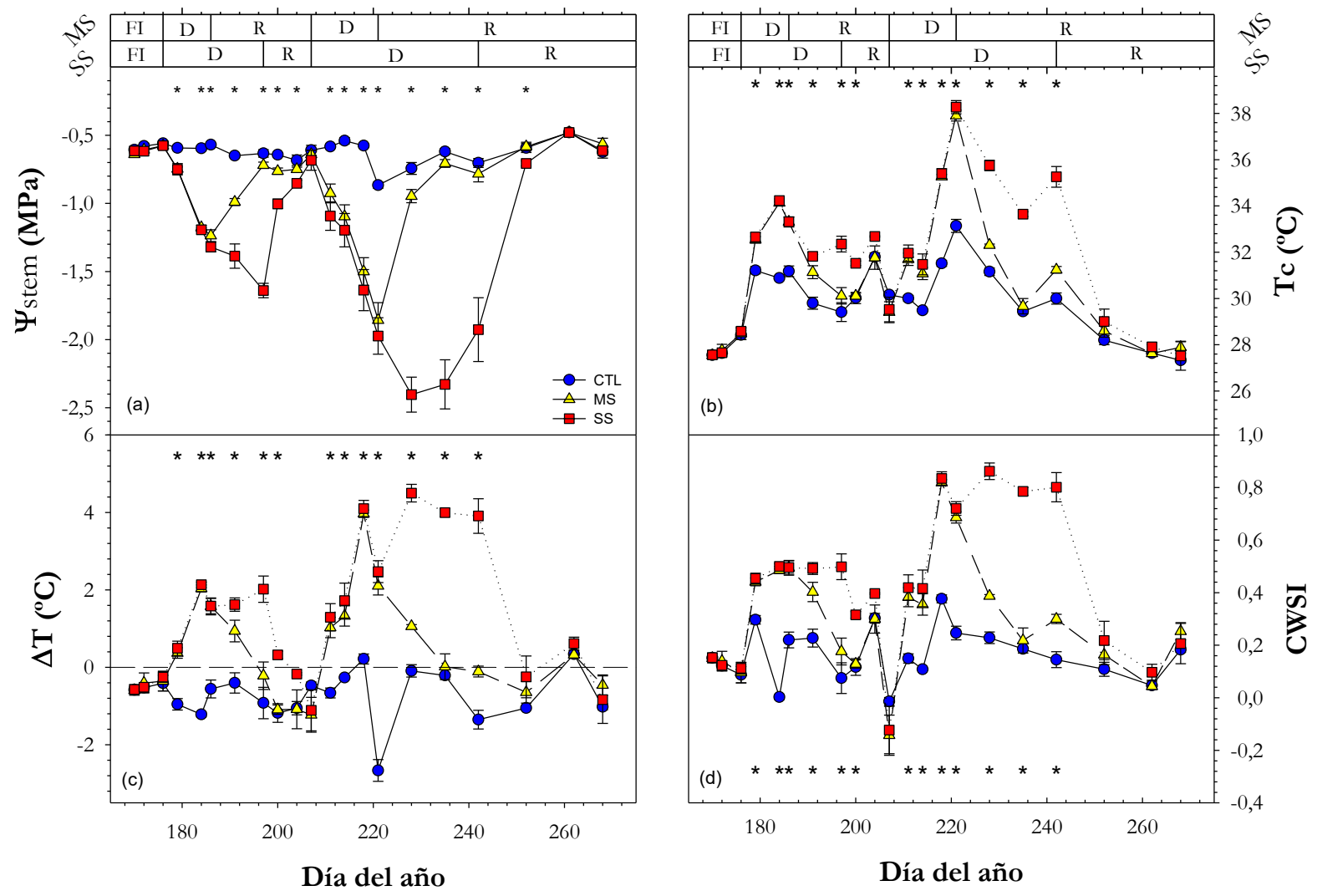

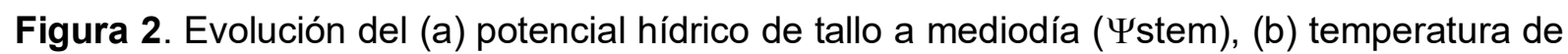
la cubierta vegetal (Tc), su diferencial con respecto al aire $(\Delta T)$ y $(c)$ del índice de estrés hídrico del cultivo (CWSI) durante el periodo de estudio. Cada punto es la media \pm ES de 6 medidas por tratamiento. Los asteriscos indican diferencias significativas según el test de Rango Múltiple de Duncan $(p<0,05)$. Las barras horizontales superiores indican los periodos de los ciclos supresión-recuperación del riego que definieron a los tratamientos MS y SS. 'Fl' es el periodo previo al inicio de los ciclos de supresión-recuperación, en el que ambos tratamientos fueron regados como CTL; ' $D$ ', periodo de supresión de riego; ' $R$ ', periodo de recuperación de los árboles.

La temperatura de copa, Tc, (Figura 2b) aumentó en los tratamientos deficitarios, posiblemente como consecuencia de la regulación estomática ante el déficit hídrico, lo que pudo reducir la transpiración y con ello la disipación de calor a través de la evaporación de agua (Jones, 2018). Este aumento de Tc se tradujo en valores $\Delta T$ superiores a los de CTL (Figura 2c), y estuvieron próximos a los valores de LLS $\left(\Delta \mathrm{T}_{\text {dry }} \approx 6,0{ }^{\circ} \mathrm{C}\right)$. Igualmente, el aumento de Tc respecto a la temperatura del aire circundante (Ta) incrementó los valores de CWSI (Figura 2d), alcanzando valores cercanos a 0,5 durante el primer ciclo de supresión y 
de 0,8 - 0,9 durante el segundo periodo de supresión en ambos tratamientos de riego deficitario. Adicionalmente se observó que los valores de CWSI alcanzados durante la segunda supresión del riego en MS fueron superiores a los obtenidos en el primer ciclo de supresión del riego de SS, lo que posiblemente se debiera a un aumento de la demanda climática reinante ( $\mathrm{ET}_{0}$ y Ta) durante este segundo periodo, además de un incremento de las necesidades hídricas del cultivo propiciado por el crecimiento vegetativo (datos no mostrados). Por otro lado, los árboles CTL mantuvieron valores inferiores a la temperatura del aire durante todo el periodo experimental $\left(\Delta \mathrm{T}\right.$ media $\left.=-0,72^{\circ} \mathrm{C}\right)$, consecuencia de una óptima tasa transpirativa. Sin embargo, los árboles CTL mostraron un valor medio de CWSI de 0,16 durante el experimento, lo que puede ser indicativo de que su apertura estomática no se correspondiese con la máxima teórica, posiblemente debido a un aumento de la resistencia al flujo del agua, aun cuando el agua del suelo no fuera un factor limitante (Jackson et al., 1988).

La Figura 3a representa la relación no lineal obtenida entre $\Psi$ stem y $\Psi \mathrm{m}$. En ella se puede observar que para valores inferiores a $-600 \mathrm{kPa}$ para MS y $-300 \mathrm{kPa}$ para SS, Ystem disminuye en mayor medida en respuestas a las reducciones de $\Psi \mathrm{m}$, este valor correspondería a un $\Psi$ stem próximo a -1,0 MPa en ambos tratamientos. Una relación similar fue descrita por Livellara et al. (2011) en cerezos 'Brooks', indicando que el estado hídrico del suelo influyó de forma evidente en el estado hídrico de la planta. Sin embargo, la relación entre $\Psi$ stem y $\Psi \mathrm{m}$ no siempre está estrechamente relacionados debido a los procesos de absorción o desorción (Blanco et al., 2018), además de una disminución en el contacto raízsuelo debido a la aparición de una fase gaseosa a medida de que el suelo se seca (Dodd et al., 2010). Por otro lado, la relación encontrada entre $\Delta \mathrm{T}$ y $\Psi \mathrm{m}$ muestra una tendencia lineal (Figura $3 \mathrm{~b}$ ), en la que disminuciones de $\Psi \mathrm{m}$ conllevan aumentos de $\Delta \mathrm{T}$.
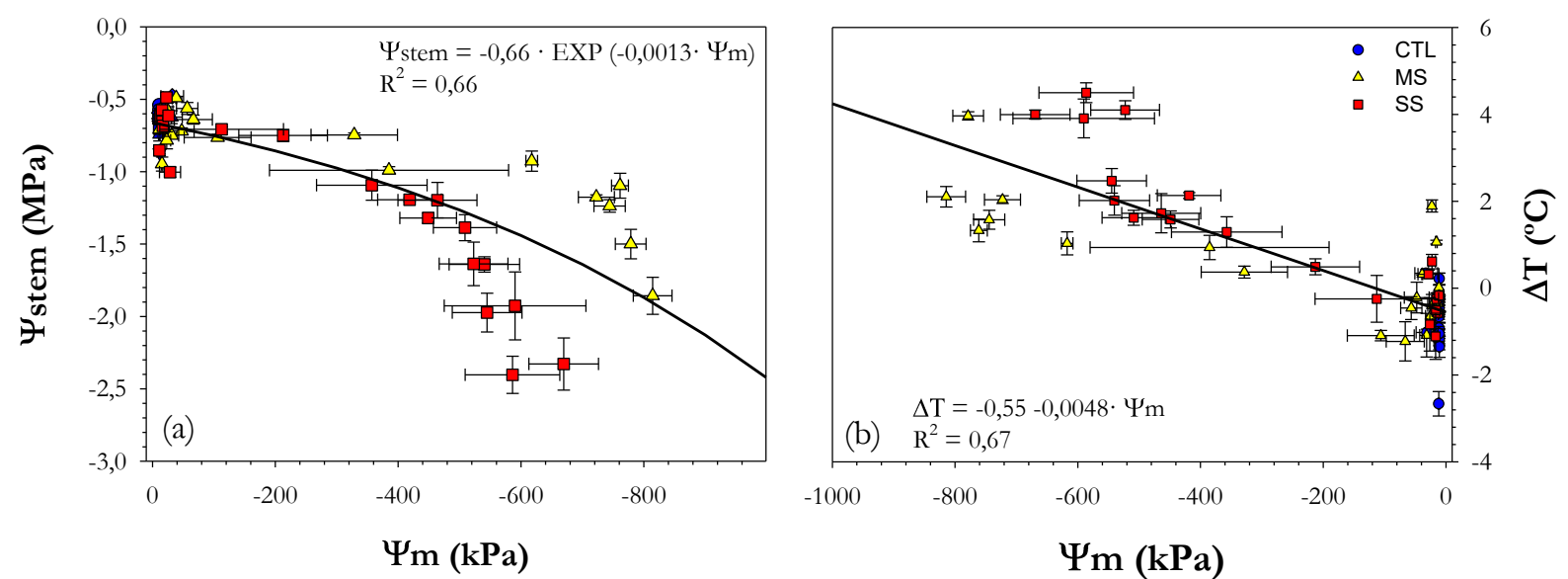

Figura 3. (a) Relación entre el potencial hídrico de tallo ( $\Psi$ stem) y el potencial matricial ( $\Psi \mathrm{m})$, y (b) relación entre la diferencia de temperatura de la copa-aire $(\Delta T)$ y $\Psi \mathrm{m}$. Cada punto es la media \pm ES de 3 medidas para $\Psi \mathrm{m}$ y 6 medidas para $\Psi$ stem y $\Delta T$ por tratamiento.

Al igual que para la relación $\Psi$ stem vs $\Psi m$ (Figura 3a), se encontró una relación no lineal entre $\Psi$ stem y Tc (Figura 4a), en la que se pueden observar dos partes diferenciadas, una más plana para altos valores de $\Psi$ stem y otra lineal de pendiente negativa a partir de valores $\Psi$ stem inferiores a $-1,0 \mathrm{MPa}$ y de temperatura superiores a $32^{\circ} \mathrm{C}$. Este segundo tramo podría estar causado por la reducción de la transpiración y la influencia de las variables meteorológicas sobre el aumento de Tc y la recarga parcial de las capacitancias del árbol durante la noche. Contrariamente a lo observado por García-Tejero et al. (2018b) en almendros, Tc muestra cierta limitación para evaluar el estado hídrico de cerezos en aquellos periodos donde el déficit hídrico es considerado como ligero-moderado. Por otro lado, se 
puede observar que las relaciones obtenidas entre $\Psi$ stem y los índices térmicos derivados de $\mathrm{Tc}, \Delta \mathrm{T}$ y CWSI, no se vieron afectadas hasta que se alcanzaron valores superiores a $0{ }^{\circ} \mathrm{C}$ para $\Delta \mathrm{T}$ y 0,25 para CWSI, momento en el cual se observó un cambio de pendiente, provocando que un descenso de $\Psi$ stem implicara un aumento proporcional de ambos índices. Estas relaciones indican que los índices calculados, de acuerdo con Jackson et al. (1977) y Jackson et al. (1981), permiten mitigar en parte la influencia de las variables meteorológicas sobre Tc en cerezos. Wang y Gartung (2010) observaron una relación lineal entre $\Psi$ stem y $\Delta T$ en peral, donde $\Psi$ stem alcanzó valores cercanos a -2,0 MPa sin que esta se viera afectada; sin embargo, Ballester et al. (2013) indicaron un cambio de tendencia de esta relación en caqui para un valor $\Psi$ stem próximo a -1,6 MPa. Wang y Gartung (2010) en peral y Tekelioğlu et al. (2017) en soja señalaron la robustez de los índices derivados de Tc en para ser utilizados en la determinación del estado hídrico de la planta en climas mediterráneos y, por tanto, como un instrumento apto para la elaboración de programas de riego.
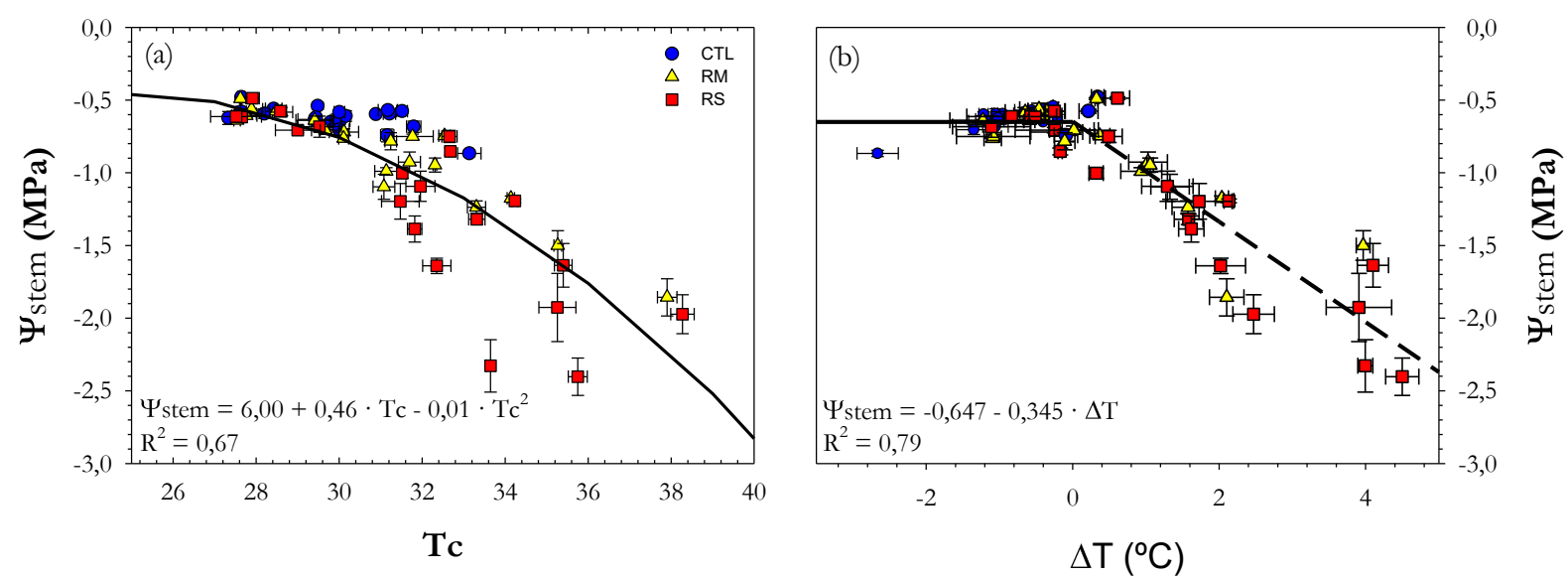

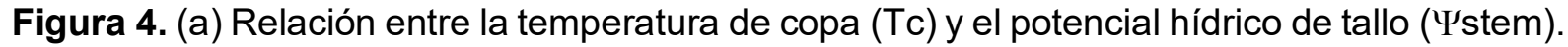
(b) Relación entre la diferencia de temperatura copa-aire $(\Delta T)$ y $\Psi$ stem. Cada punto es la media \pm ES de 6 medidas por tratamiento.

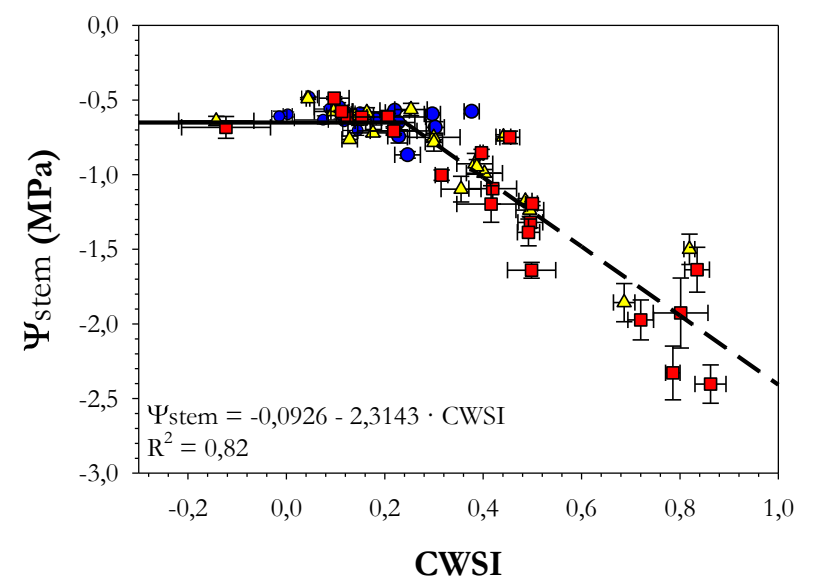

Figura 5. Relación entre el índice de estrés hídrico del cultivo (CWSI) y el potencial hídrico de tallo (Ystem). Cada punto es la media \pm ES de 6 medidas por tratamiento.

Durante el periodo experimental, se observó que el crecimiento vegetativo, determinado a partir del incremento del diámetro de tronco, $\Delta \mathrm{DT}$, y del peso de la poda, fue 
sensible al régimen hídrico impuesto durante el periodo de estudio (Figura 6), observándose reducciones de $\triangle \mathrm{DT}$ con respecto a CTL de $24 \%$ y $41 \%$, para MS y SS, respectivamente. Por otro lado, la poda mostró una sensibilidad similar al déficit hídrico que $\Delta \mathrm{DT}$, obteniendo reducciones del $38 \%$ y $64 \%$ con respecto a los árboles de CTL para MS y SS, respectivamente. Estos datos confirman los obtenidos por Nieto et al. (2017), donde encontraron una relación positiva entre el crecimiento vegetativo de los árboles y el volumen de agua aplicado. Además, otros autores han reportado que un déficit hídrico en árboles jóvenes puede afectar drásticamente al volumen de copa y a su entrada en producción (Livellara et al., 2011).
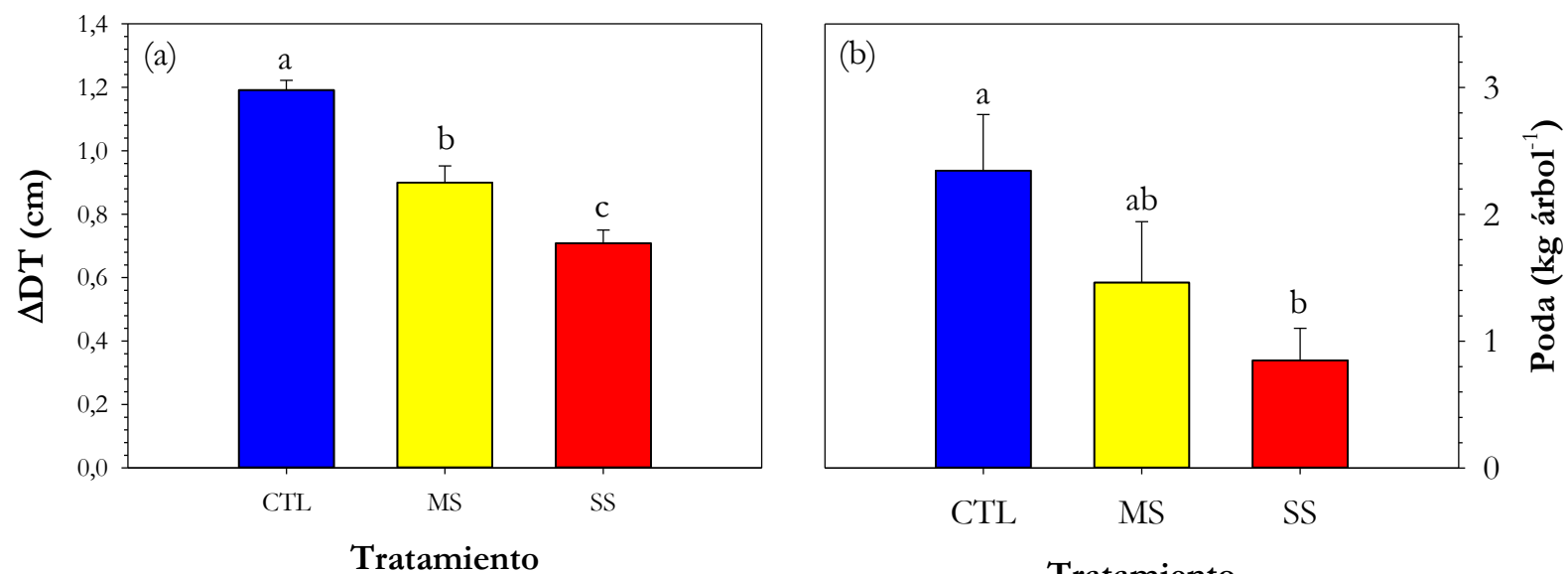

Tratamiento

Figura 6. (a) Variación del diámetro de tronco $(\Delta \mathrm{DT})$ y peso de poda correspondiente al periodo de estudio. Cada punto es la media \pm ES de 6 medidas por tratamiento. Barra con letras diferentes indican diferencias significativas según el test de Rango Múltiple de Duncan $(p<0,05)$.

\section{5) Conclusiones}

Im mostró una rápida respuesta al cambio del contenido de agua del suelo. Sin embargo, presentó una variabilidad superior a la de los indicadores de planta estudiados, especialmente respecto a $\Psi$ stem, lo que sugiere la necesidad de disponer de un mayor número de medidas para representar adecuadamente el estado hídrico del suelo e indirectamente del cultivo a través de $\Psi \mathrm{m}$. Por otro lado, el uso de la temperatura de copa a través de sus índices $\Delta T$ y CWSI puede ser muy útil para estudiar y evaluar la respuesta de cerezos al déficit hídrico, así como para elaborar y supervisar programas de riego basados en niveles de estrés diferentes. El estrés hídrico de los árboles MS y SS afectó de forma clara al crecimiento del árbol, provocando reducciones en el peso de poda del 40 y $60 \%$, respectivamente; indicando la alta sensibilidad de este proceso al déficit hídrico en plantaciones jóvenes.

\section{6) Agradecimientos}

Este trabajo fue financiado por el Ministerio de Economía, Industria y Competitividad del Gobierno de España, MICIIN (Ref. AGL2016-77282-C3-3-R) y la Fundación Séneca de la Región de Murcia (Grupo de excelencia 2016-2019, Ref. 19895/GERM/15). 


\section{6) Bibliografía}

Allen, R.G., Pereira, L.S., Raes, D., Smith, M., 1998. Crop evapotranspiration. Guidelines for computing crop water requirements. FAO Irrigation and Drainage paper No. 56. Rome, Italy, pp 15-27.

Ballester, C., Jiménez-Bello, M.A., Castel, J.R., Intrigliolo, D.S., 2013. Usefulness of thermography for plant water stress detection in citrus and persimmon trees. Agric. For. Meteorol. 168, 120-129.

Blanco, V., Domingo, R., Pérez-Pastor, A., Blaya-Ros, P.J., Torres-Sánchez, R., 2018. Soil and plant water indicators for deficit irrigation management of fieldgrown sweet cherry trees. Agric. Water Manag. 208, 83-94.

Blanco, V., Torres-Sánchez, R., Blaya-Ros, P.J., Pérez-Pastor, A., Domingo, R., 2019. Vegetative and reproductive response of 'Prime Giant' sweet cherry trees to regulated deficit irrigation. Sci. Hortic. (Amsterdam). 249, 478-489.

Costa, J.M., Grant, O.M., Chaves, M.M., 2013. Thermography to explore plantenvironment interactions. J. Exp. Bot. 64, 3937-3949.

Dodd, I.C., Egea, G., Watts, C.W., Whalley, W.R., 2010. Root water potential integrates discrete soil physical properties to influence ABA signalling during partial rootzone drying. J. Exp. Bot. 61, 3543-3551.

Fereres, E., Castel, J.R., 1981. Drip Irrigation Management. Division of Agricultural Sciences, University of California (Leaflet 21259).

Fereres, E., Soriano, M.A., 2007. Deficit irrigation for reducing agricultural water use. J. Exp. Bot. 58, 147-159.

García-Tejero, I.F., Gutiérrez-Gordillo, S., Ortega-Arévalo, C., Iglesias-Contreras, M., Moreno, J.M., Souza-Ferreira, L., Durán-Zuazo, V.H., 2018a. Thermal imaging to monitor the crop-water status in almonds by using the non-water stress baselines. Sci. Hortic. (Amsterdam). 238, 91-97.

García-Tejero, I.F., Gutiérrez-Gordillo, S., Souza, L., Cuadros-Tavira, S., DuránZuazo, V.H., 2018b. Fostering sustainable water use in almond (Prunus dulcis Mill.) orchards in a semiarid Mediterranean environment. Arch. Agron. Soil Sci. 65, 164-181.

García-Tejero, I.F., Ortega-Arévalo, C.J., Iglesias-Contreras, M., Moreno, J.M., Souza, L., Tavira, S.C., Durán-Zuazo, V.H., 2018c. Assessing the crop-water status in almond (Prunus dulcis Mill.) trees via thermal imaging camera connected to smartphone. Sensors 18, 1050.

Gómez-Bellot, M.J., Nortes, P.A., Sánchez-Blanco, M.J., Ortuño, M.F., 2015. Sensitivity of thermal imaging and infrared thermometry to detect water status changes in Euonymus japonica plants irrigated with saline reclaimed water. Biosyst. Eng. 133, 21-32.

Idso, S.B., Jackson, R., Pinter, P.J., Reginato, R.J., Hatfield, J.L., 1981. Normalizing the stress-degree-day parameter for environmental variability. Agric. Meteorol. $24,45-55$.

Jackson, R.., Idso, S.., Reginato, R.., Pinter, P.., 1981. Canopy temperature as a Crop Water Stress Indicator. Water Resour. Res. 17, 1133-1138.

Jackson, R.D., Kustas, W.P., Choudhury, B.J., 1988. A reexamination of the crop water stress index. Irrig. Sci. 9, 309-317.

Jackson, R.D., Reginato, R.J., Idso, S.B., 1977. Wheat canopy temperature: A practical tool for evaluating water requirements. Water Resour. Res. 13, 651656.

Jones, H.G., 2018. Thermal Imaging and Infrared Sensing in Plant Ecophysiology. In 


\begin{abstract}
A. M. Sánchez-Moreiras, A. M., Reigosa, M. J. (Eds.), Advances in Plant Ecophysiology Techniques. pp. 135-151.
\end{abstract}

Jones, H.G., 2007. Monitoring plant and soil water status: established and novel methods revisited and their relevance to studies of drought tolerance. J. Exp. Bot. 58, 119-130.

Jones, H.G., 1999. Use of infrared thermometry for estimation of stomatal conductance as a possible aid to irrigation scheduling. Agric. For. Meteorol. 95, 139-149.

Livellara, N., Saavedra, F., Salgado, E., 2011. Plant based indicators for irrigation scheduling in young cherry trees. Agric. Water Manag. 98, 684-690.

Marsal, J., 2012. FAO irrigation and drainage paper 66. Crop Yield Response Water. Sweet Cherry. FAO, Rome, pp. 449-457.

Marsal, J., Lopez, G., del Campo, J., Mata, M., Arbones, A., Girona, J., 2010. Postharvest regulated deficit irrigation in "Summit" sweet cherry: Fruit yield and quality in the following season. Irrig. Sci. 28, 181-189.

Naor, A., Gal, Y., Peres, M., 2006. The inherent variability of water stress indicators in apple, nectarine and pear orchards, and the validity of a leaf-selection procedure for water potential measurements. Irrig. Sci. 24, 129-135.

Nieto, E., Prieto, M.H., Fortes, R., Gonzalez, V., Campillo, C., 2017. Response of a long-lived cherry cultivar to contrasting irrigation strategies in the Jerte Valley, Extremadura, Spain. Acta Hortic. 1161, 197-204.

Schackel, K.A., Ahmadi, H., Biasi, W., Burchner, R., Goldhamer, D.A., Gurusinghe, S., Hasey, J., Kester, D., Krueger, B., Lampinen, B., McGourty, G., Micke, W., Mitcham, E., Olson, B., Pelletrau, K., Philips, H., Ramos, D., Schwankl, L., Sibbett, S., Snyder, R., Southwick, S., Stevenson, M., Thorpe, M., Weinbaum, S., Yeager, J., 1996. Plant water status as an index for irrigation need in deciduos fruit tress. Hort Technol. 7, 23-29.

Tekelioğlu, B., Büyüktaş, D., Baştuğ, R., Karaca, C., Aydinşakir, K., Dinç, N., 2017. Use of Crop Water Stress Index for Irrigation Scheduling of Soybean in Mediterranean Conditions. J. Exp. Agric. Int. 18, 1-8.

Wang, D., Gartung, J., 2010. Infrared canopy temperature of early-ripening peach trees under postharvest deficit irrigation. Agric. Water Manag. 97, 1787-1794. 